\title{
Clinical implication of MEN1 mutation in surgically resected thymic carcinoid patients
}

\author{
Xiongfei $\mathrm{Li}^{1 *}$, Mingbiao $\mathrm{Li}^{1 *}$, Tao Shi ${ }^{2 *}$, Renwang Liu ${ }^{1}$, Dian $\operatorname{Ren}^{1}$, Fan Yang $^{1}$, Sen Wei ${ }^{1}$, Gang Chen ${ }^{1}$, Jun \\ Chen $^{1,3}$, Song $\mathrm{Xu}^{1,3}$ \\ ${ }^{1}$ Department of Lung Cancer Surgery, ${ }^{2}$ Department of Pathology, ${ }^{3}$ Tianjin Key Laboratory of Lung Cancer Metastasis and Tumor \\ Microenvironment, Lung Cancer Institute, Tianjin Medical University General Hospital, Tianjin 300052, China \\ *These authors contributed equally to this work. \\ Correspondence to: Jun Chen, MD, PhD and Song Xu, MD, PhD. Department of Lung Cancer Surgery, Lung Cancer Institute, Tianjin Medical University \\ General Hospital, No. 154 Anshan Road, Heping District, Tianjin 300052, China. Email:huntercj2004@yahoo.com; xusong198@hotmail.com.
}

\begin{abstract}
Thymic carcinoid is a rare but very aggressive neuroendocrine tumour derived from the neuroendocrine system. Here we report a male patient with thymic atypical carcinoid. Though thymic carcinoid is relatively common, the gene sequencing profile was performed and the gene sequencing result indicated germline multiple endocrine neoplasia type 1 (MEN1) mutation and two somatic mutations on MEN1 gene and no copy number variation or fusion events were detected. It is well-known that the mutation of MEN1 is the typical manifestation of MEN1 syndrome, which is an autosome dominant disease that includes varying combinations of more than 20 endocrine and non-endocrine tumors. In the English literature, 7 cases of solitary thymic carcinoid harboring somatic variants in MEN1 are reported in the absence of other organs involvement as MEN1 syndrome presents. We summarized the clinical features and prognosis of this rare thymic tumor.
\end{abstract}

Keywords: Multiple endocrine neoplasia type 1 (MEN1); thymic carcinoid; next generation sequencing (NGS); prognosis

Submitted Sep 20, 2017. Accepted for publication Jan 17, 2018.

doi: $10.21037 /$ jtd.2018.01.127

View this article at: http://dx.doi.org/10.21037/jtd.2018.01.127

\section{Introduction}

Thymic carcinoid refers to a neuroendocrine tumour arising in the thymus, accounting for about $2-7 \%$ of anterior mediastinal masses $(1,2)$. Approximately $40 \%$ of patients have Cushing syndrome as a result of adrenocorticotropic hormone secretion by the tumour (1). Since the initial report of thymic carcinoid by Rosai and Higa in 1972 (3), about 200 cases of thymic carcinoid have been reported in English literature so far. We describe a 62-year-old male patient with surgically resected thymic carcinoid and mutations of MEN1 gene were detected by next generation sequencing (NGS). Previous evidence indicates that thymic carcinoids occur in $1-5 \%$ of patients with multiple endocrine neoplasia type 1 (MEN1) syndrome and are a major cause of morbidity and mortality (4). We did a literature review and analyzed the clinicopathologic characteristics of all thymic carcinoid patients with the initial manifestation of MEN1 syndrome. All of thymic carcinoids patients with somatic mutation of MEN1 gene had MEN1 syndrome. Considering none of other organs involved and a negative family history of MEN1 syndrome, our patient could not be diagnosed with MEN1 syndrome at this moment. Based on the findings of our literature review, we proposed that somatic mutation test of $M E N 1$ gene and evaluation of other organs are recommended for thymic carcinoid patients. Moreover, close follow-up of thymic carcinoid patients with somatic mutation of MEN1 gene is necessary and could help detect disease recurrence and metastases early.

\section{Case presentation}

A 62-year-old male patient was admitted to the hospital 
A
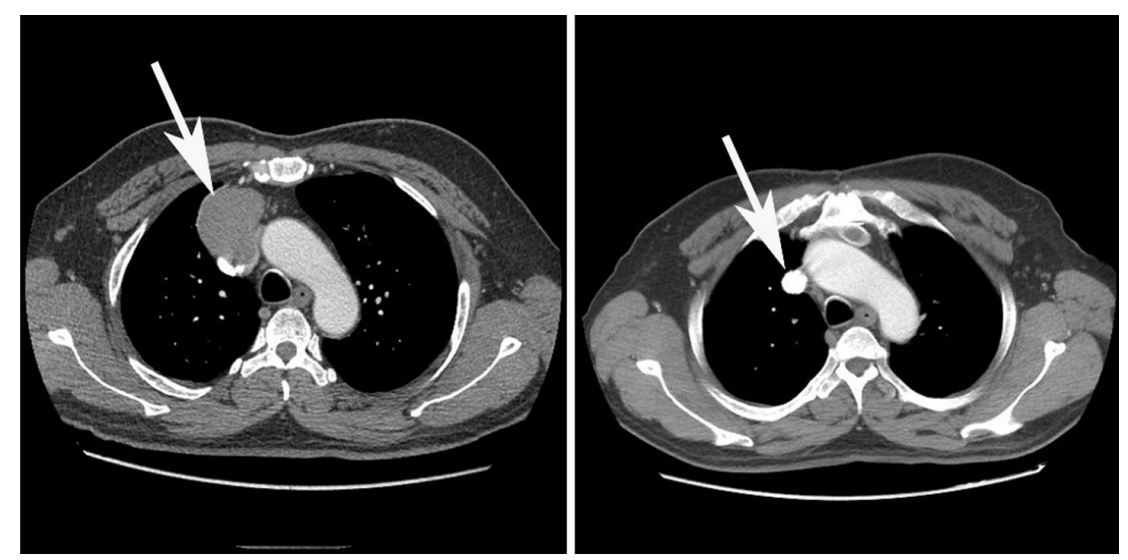

\section{B}
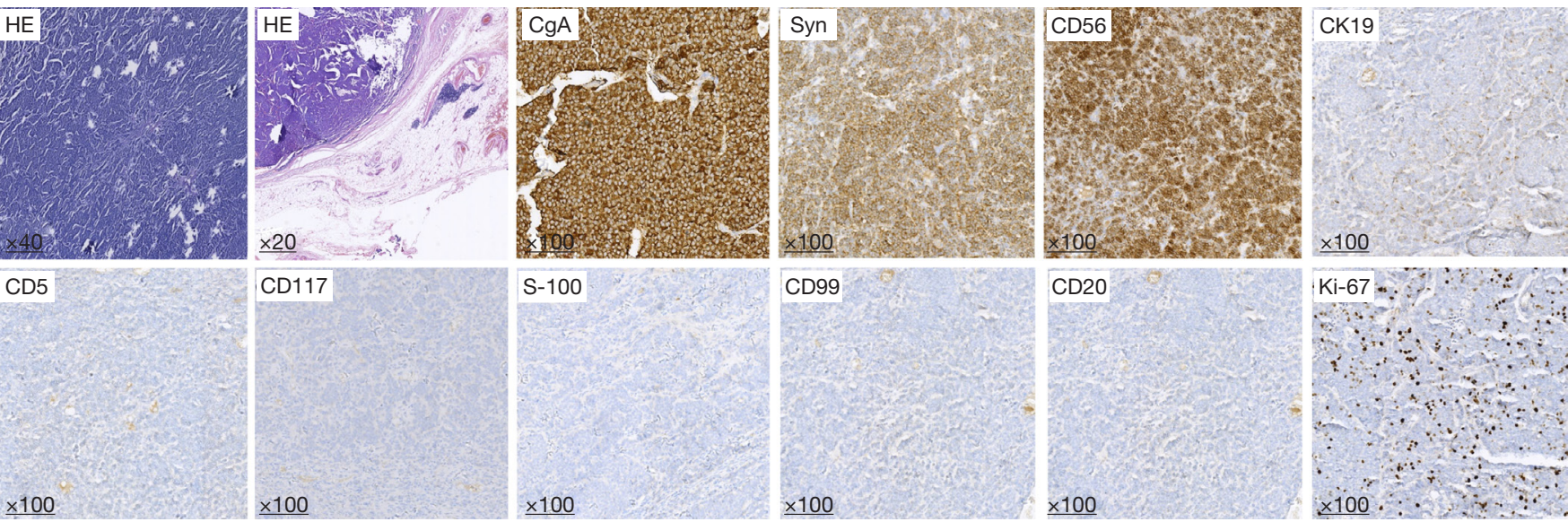

CD117
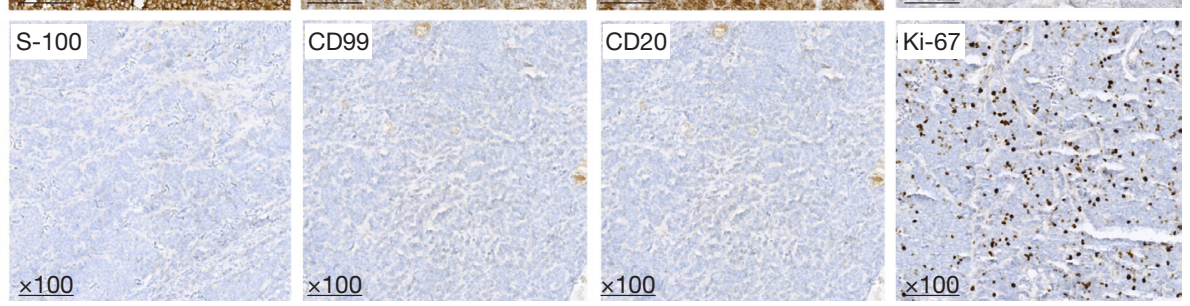

Figure $1 \mathrm{CT}$ images and pathological stainings. (A) Left: preoperative CT image. Arrow indicates that anterior mediastinum invades the superior vena cava. Right: postoperative CT image. Arrow indicates artificial blood vessel reconstruction; (B) HE and IHC stainings. CT, computed tomography; HE, hematoxylin and eosin; IHC, immunohistochemistry; CgA, chromogranin A.

because of chest discomfort in June 2015. An enhanced chest computed tomography (CT) showed an anterior mediastinum mass invading superior vena cava, approximately $6 \mathrm{~cm} \times 5 \mathrm{~cm} \times 2 \mathrm{~cm}$ in size (Figure 1A). Positron emission tomography indicated the mass had an abnormal $18 \mathrm{~F}$ fludeoxyglucose (FDG) uptake and a preliminary diagnosis of malignant anterior mediastinum mass was given. Physical examination, laboratory evaluation, and radiological tests of other organs revealed no significant abnormalities. A resection of mediastinum mass together with systemic lymphadenectomy and artificial blood vessel reconstruction was performed using a midline approach through a sternotomy. Macroscopically, the mass was $5.5 \mathrm{~cm} \times 4.5 \mathrm{~cm} \times 2.2 \mathrm{~cm}$ with gray, soft and smooth section. Microscopically, postoperative hematoxylin and eosin (HE) staining showed thymic tissue, together with 2-4 nuclear mitosis per $10 \mathrm{HPF}$ and focal necrosis. And immunohistochemistry (IHC) staining was positive for chromogranin A (CgA), Syn, CD56, CK19 and negative for CD5, CD117, S-100, CD99, CD20 with a Ki-67 index of $15 \%$ (Figure $1 B$ ). Through HE and IHC stainings, the diagnosis of thymic atypical carcinoid was established. Postoperative CT showed clear lungs and artificial blood vessels with smooth blood flow (Figure 1A). The patient recovered well and was discharged favorably. The patient refused to receive any postoperative therapy except close follow-up. Until 20 months postoperatively, the patient was symptom-free and had no evidences of local and systematic abnormalities.

To explore the underlying mechanisms, a genetic mutation profiling of 295 cancer related genes was performed by NGS (OncoScreenTM 295 genes, Burning Rock Dx, Guangzhou, China, Figure S1). The gene sequencing result indicated germline MEN1 mutation and two somatic mutations on MEN1 gene (One nonsense mutation in exon 10 with $45.6 \%$ frequency and one 


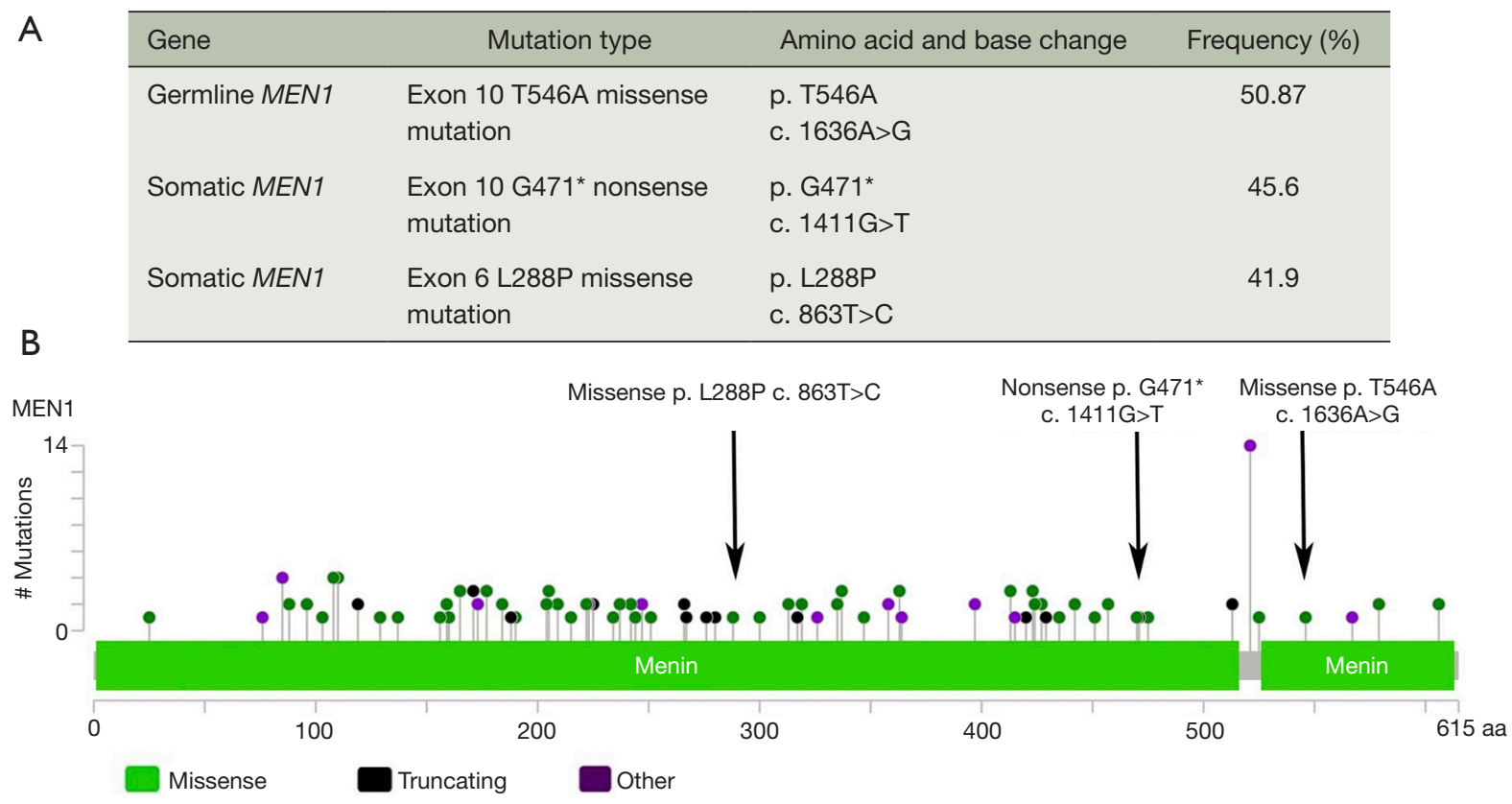

Figure 2 Gene mutation analysis. (A) NGS analysis detected germline and somatic MEN1 mutations; (B) MutationMap of MEN1 mutation from TCGA database. NGS, next generation sequencing; MEN1, multiple endocrine neoplasia type 1; TCGA, the cancer genome atlas.

missense mutation in exon 6 with $41.9 \%$ frequency) and no copy number variation or fusion events were detected (Figure 2A). We further point out somatic mutation locus of our patient on MutationMap together with all MEN1 mutations from the cancer genome atlas (TCGA) database (Figure 2B). We also retrieved the clinicopathologic characteristics of all thymic carcinoid patients with the initial manifestation of MEN1 syndrome in previous published literatures from PubMed.

\section{Discussion}

MEN1 gene mutations can be identified in $70-95 \%$ of MEN1 patients. MEN1 follows Knudson's "two-hit" model for tumor suppressor gene carcinogenesis. The first hit is a heterozygous MEN1 germline mutation, and the second hit is a MEN1 somatic mutation and gives cells the survival advantage needed for tumor development (5). The thymic carcinoid of our case might be caused by the twice mutations on MEN1 gene according to Knudson's "two-hit" model. Carcinoid tumors are estimated to occur in about $10 \%$ of MEN1 patients, and thymic carcinoids were reported be more aggressive with a poor prognosis of 10 -year overall survival rate about $25-36 \%$ in the literature (4). Thymic carcinoids are more prevalent in males than in females and most patients are clinically silent.

In literature, we found six patients exhibited thymic carcinoid as the initial manifestation of MEN1 syndrome $(4,6-10)$. We summarized the clinical features of these cases (Table 1). We confirmed that a higher percentage of thymic carcinoids can occur in men which might the effect of sex hormone promoting the proliferation and maturation of thymocyte in MEN1 syndrome (Table 1) (11). The age of patients was young, ranging from 23 to 53 with a median age is 36.5 , which might indicate the aggressive nature of disease. Gibril et al. reported that about 1/3 MEN1 related thymic carcinoid patients were asymptomatic or only have non-specific symptoms when they had already local invasion or metastases at the time of initial diagnosis (12). The mutations of MEN1 are also listed in the Table 1. The types of mutations include duplication, insertion, deletion, frameshift and nonsense mutation, which occur in exon 2, 3, 5, 9 and 10. Among all the mutations, the mutations in exon10 occur three times which is the same for our case.

Although MEN1 related thymic carcinoid patients only account for less than 5 percentage of all the MEN1 patients, they were associated with an increased mortality and a poorer prognosis due to the more aggressive nature and potential for metastasis (13-15). Early and correct diagnosis is particularly important for thymic carcinoid patients with 
Table 1 Literature review of patients with thymic carcinoid as the initial manifestation of MEN1 syndrome

\begin{tabular}{|c|c|c|c|c|c|c|c|c|}
\hline Reference & Age/sex & Smoking & $\begin{array}{l}\text { Type of MEN1 } \\
\text { mutation }\end{array}$ & $\begin{array}{l}\text { Other organs } \\
\text { involvement }\end{array}$ & $\begin{array}{l}\text { Family history } \\
\text { of MEN1 }\end{array}$ & $\begin{array}{l}\text { MEN1 syndrome } \\
\text { diagnosis }\end{array}$ & $\begin{array}{l}\text { Follow-up } \\
\text { (months) }\end{array}$ & Status \\
\hline $\begin{array}{l}\text { Christakis } \\
\text { et al. (4) }\end{array}$ & $23 / \mathrm{M}$ & No & $\begin{array}{l}\text { Duplication and } \\
\text { insertion mutation } \\
\text { of exons } 9,10\end{array}$ & None & NK & Yes & 84 & Dead \\
\hline $\begin{array}{l}\text { Hasani-Ranjbar } \\
\text { et al. (6) }\end{array}$ & 29/M & No & $\begin{array}{l}\text { Deletion mutation } \\
\text { exon } 10\end{array}$ & $\mathrm{PH} / \mathrm{PA}$ & Yes & Yes & 24 & Alive \\
\hline Ghazi et al. (8) & $44 / \mathrm{M}$ & NK & $\begin{array}{l}\text { Frameshift mutation } \\
\text { exon } 10\end{array}$ & $\mathrm{PA} / \mathrm{PH}$ & Yes & Yes & 49 & Dead \\
\hline Ferolla et al. (9) & $47 / \mathrm{M}$ & Yes & $\begin{array}{l}\text { Frameshift mutation } \\
\text { exon } 2\end{array}$ & $\mathrm{PH}$ & Yes & Yes & 36 & Dead \\
\hline
\end{tabular}

M, male; F, female; NK, not known; PH, parathyroid hyperplasia; PA, pituitary adenoma; PET, pancreatic endocrine tumor; MEN1, multiple endocrine neoplasia type 1.

MEN1 syndrome. In our case, the patient was diagnosed as thymic atypical carcinoid with mutation of MEN1 gene. But he had none of other organs involvement at the time of diagnosis and a negative family history of MEN1 syndrome. However, based on the findings of our literature review, we have to bear in mind that this thymic carcinoid patient with MEN1 somatic mutation might develop metachronous neoplasias.

Therefore, we proposed that gene mutation test of MEN1 and evaluation of other organs are recommended for thymic carcinoid patients. Moreover, close follow-up of thymic carcinoid patients with somatic mutation of MEN1 gene is necessary and could help detect disease recurrence and metastases early.

\section{Conclusions}

Thymic carcinoid patients with MEN1 gene mutation are rare. Literature review shows that a much higher percentage of MEN1 gene mutation related thymic carcinoids can occur in men and develop synchronous or metachronous neoplasia in other organs. Therefore, for patients with thymic carcinoid, testing of MEN1 gene mutation is recommended. In addition, systematic evaluations and close follow-up are necessary for the thymic carcinoid patients with MEN1 mutation because of the highly possible involvement of other organs and development of MEN1 syndrome.

\section{Acknowledgements}

Funding: This work was financially supported by grants from the National Natural Science Foundation of China (81772464, 81773207), the Science and Technology Support Key Program of Tianjin (17YFZCSY00840), Tianjin Key Project of Natural Science Foundation (16JCZDJC34200, 16PTSYJC00160, 17JCZDJC36200).

\section{Footnote}

Conflicts of Interests: The authors have no conflicts of interest to declare.

Informed Consent: The patient granted written informed consent for publication of this manuscript and the accompanying images.

\section{References}

1. Recuero Díaz JL, Embún Flor R, Menal Muñoz P, et al. Thymic carcinoid associated with multiple endocrine neoplasia syndrome type I. Arch Bronconeumol 2013;49:122-5.

2. Dixon JL, Borgaonkar SP, Patel AK, et al. Thymic neuroendocrine carcinoma producing ectopic adrenocorticotropic hormone and Cushing's syndrome. Ann Thorac Surg 2013;96:e81-3. 
3. Rosai J, Higa E. Mediastinal endocrine neoplasm, of probable thymic origin, related to carcinoid tumor. Clinicopathologic study of 8 cases. Cancer 1972;29:1061-74.

4. Christakis I, Qiu W, Silva Figueroa AM, et al. Clinical Features, Treatments, and Outcomes of Patients with Thymic Carcinoids and Multiple Endocrine Neoplasia Type 1 Syndrome at MD Anderson Cancer Center. Horm Cancer 2016;7:279-87.

5. Marx SJ, Agarwal SK, Kester MB, et al. Germline and somatic mutation of the gene for multiple endocrine neoplasia type 1 (MEN1). J Intern Med 1998;243:447-53.

6. Hasani-Ranjbar S, Rahmanian M, Ebrahim-Habibi A, et al. Ectopic Cushing syndrome associated with thymic carcinoid tumor as the first presentation of MEN1 syndrome-report of a family with MEN1 gene mutation. Fam Cancer 2014;13:267-72.

7. Kikuchi R, Mino N, Okamoto T, et al. Simultaneous double thymic carcinoids: a rare initial manifestation of multiple endocrine neoplasia type 1 . Gen Thorac Cardiovasc Surg 2011;59:68-72.

8. Ghazi AA, Dezfooli AA, Mohamadi F, et al. Cushing syndrome secondary to a thymic carcinoid tumor due to multiple endocrine neoplasia type 1. Endocr Pract 2011;17:e92-6.

Cite this article as: $\mathrm{Li} \mathrm{X}$, Li M, Shi T, Liu R, Ren D, Yang F, Wei S, Chen G, Chen J, Xu S. Clinical implication of MEN1 mutation in surgically resected thymic carcinoid patients. J Thorac Dis 2018;10(2):E125-E129. doi: 10.21037/ jtd.2018.01.127
9. Ferolla P, Falchetti A, Filosso P, et al. Thymic neuroendocrine carcinoma (carcinoid) in multiple endocrine neoplasia type 1 syndrome: the Italian series. J Clin Endocrinol Metab 2005;90:2603-9.

10. Boix E, Picó A, Pinedo R, et al. Ectopic growth hormonereleasing hormone secretion by thymic carcinoid tumour. Clin Endocrinol (Oxf) 2002;57:131-4.

11. Seiki K, Sakabe K. Sex hormones and the thymus in relation to thymocyte proliferation and maturation. Arch Histol Cytol 1997;60:29-38.

12. Gibril F, Chen YJ, Schrump DS, et al. Prospective study of thymic carcinoids in patients with multiple endocrine neoplasia type 1. J Clin Endocrinol Metab 2003;88:1066-81.

13. Ito T, Jensen RT. Imaging in multiple endocrine neoplasia type 1: recent studies show enhanced sensitivities but increased controversies. Int J Endocr Oncol 2016;3:53-66.

14. Goudet P, Murat A, Binquet C, et al. Risk factors and causes of death in MEN1 disease. A GTE (Groupe d'Etude des Tumeurs Endocrines) cohort study among 758 patients. World J Surg 2010;34:249-55.

15. Brandi ML, Gagel RF, Angeli A, et al. Guidelines for diagnosis and therapy of MEN type 1 and type 2. J Clin Endocrinol Metab 2001;86:5658-71. 
Supplementary

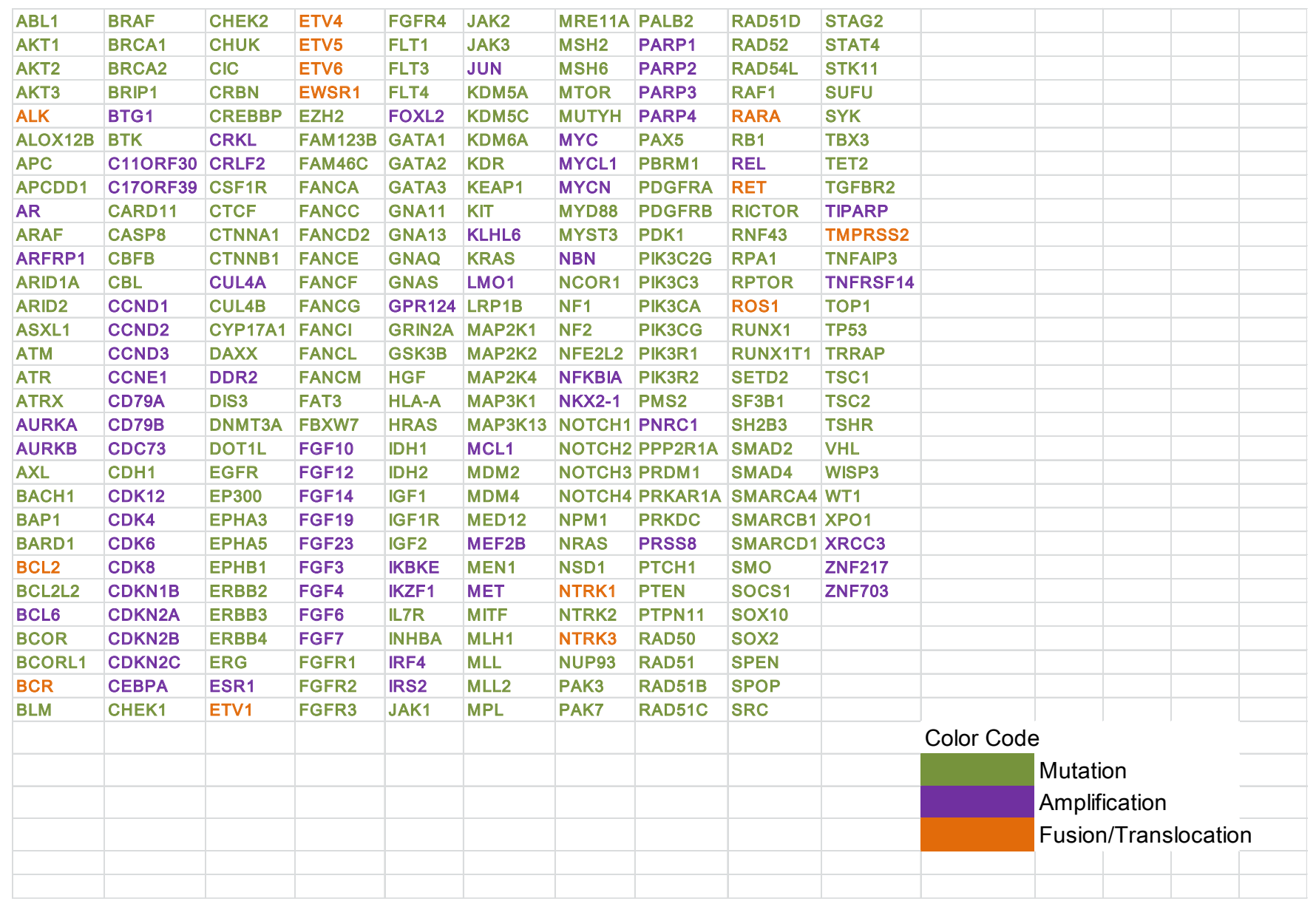

Figure S1 Next generation sequencing on the mutations of 295 tumor-related genes. 